the solid body. Contact action seems also to have played its part in the researches of $\mathbf{M}$. Lemoine on the dissociation of hydrogen iodide. On the whole, in all those cases where the process of chemical transformation in a gaseous medium offers an uninterrupted character, there is reason to suppose that a contact action has been taking place. But if this supposition proved to be correct, we should be compelled to admit that the chemical transformation, even in its simplest shape in a gaseous medium, is intimately connected with the action of molecular forces-that is, with such actions which do not have the characters of determinated chemical combinations. Molecular forces ought to be taken into account even in the transformations going on in a gaseous medium ; both factors--tbe chemical affinity and the cohesion-appear so intimately connected that it would be impossible to delimitate them : the chemical reaction would appear as a result of both the forces which unite atoms in molecules and those which are at work between the molecules.

THE last issue of the Fournal of the Russian Chemical Society (xvii. 7) contains the first part of a most valuable inquiry, by M. Konovaloff, into the part played by contact actions in dissociation. Without undertaking to deal with this immense subject in full, the author, taking advantage of observations he had made together with Prof. Menschutkin during their experiments as to the dissociation of compound ethers, has submitted to a closer investigation the contact phenomena when gases are brought into contact with solids. The want of cohesion between the gaseous molecules, and thegreat difference of densities of both the gas and the solid, give better conditions under which to study the influence of the solid. Summing up the researches of Sainte-Claire Deville, Würtz, Faraday, Ramsay, Berthelot, and many others who have devoted attention to the subject, the author shows that capillary structure and porosity are not necessary conditions in a solid body for producing dissociation: smooth surfaces may also condense vapours and gases, and sometimes retain them with such a force as to make the disengagement of the absorbed gas quite correspond to the dissociation of a chemical compound. The character of the surface, having of course a great importance, M. Konovaloff has carried on his experiments so as to study the influence of the character of the surface. The first part of his inquiry contains the experiments made as to the dissociation of the tertiary amylacetate, the method of inquiry being successive determinations of the density of its vapcurs on W. Meyer's method. The result arrived at is obviously that the structure of the surface of the glass which is brought into contact with amylacetate vapours is of great importance; but it is worthy of notice that the rough surface of the glass-powder condenses the vapour without producing a notable dissociation, while the smooth surface of the glasscotton dissociates it.

\section{THE INSTITUTION OF MECHANICAL ENGINEERS}

T'HIS Institution held its annual meeting at the theatre of the Institution of Civil Engineers on Thursday and Friday last under the presidency of Mr. Jeremiah Head, who was re-elected for the ensuing year.

A paper was read by Mr. J. H. Wicksteed descriptive of an autographic test-recording apparatus of a very ingenious character. It is designed to obviate both the labour of observation and that of hand-plotting. But, beside the saving of time and labour, there is the further gain, in obtaining the diagrams autographically, that the progress of the test is continuoits; and as time is a factor in the behaviour of a test-piece, it is important in making tests for comparison that there should be no irregularity in this factor.

The sample is held between an upper and lower gripping-box. The upper box is suspended from the back centre of a steelyard, which, by the adjustment of its poise-weight, weighs whatever pull is put upon the sample. The lower box is connested with a hydraulic cylinder, which puts the pull upon the sample, and extends it until it breaks. Thus while the hydraulic cylinder is doing the mechanical work of breaking the sample, the steelyard is measuring the load it sustains. The object of the indicator is to record simultaneously the amount of the load and the extension due to it. To get this simultaneous record the horizontal ram of the indicator, which carries the tracing pencil, is in fluid connection with the hydraulic cylinder which puts the load upon the sample, and the indicator therefore partakes of that load. Round the outer end of the ram is coiled a spiral spring, which is compressed as the pressure on the ram increases, and expands as the fluid pressure on the ram decreases; the pencil records the point of equilibrium between the two. The friction of the leathers in the hydraulic cylinder and that of the indicator ram are both eliminated from the diagram, the first by putting on to the piston of the hydraulic cylinder a gross pressure equal to the effective pressure on the sample and the friction of the hydraulic leathers, and the second by revolving the indicator ram by belt power and gearing; the driving power being applied in a plane at right angles to the longitudinal travel of the ram has no effect upon that travel, but entirely overcomes the obstruction which the friction of the leather would otherwise offer to the free travel of the ram, so that the ram becomes sensitive enough to respond to the very smallest want of balance between the opposite forces of the water pressure and the spring. For recording the extension of the sample simultaneously with the load upon it, the metallic paper on which the pencil travels is mounted on a brass barrel like that of an ordinary steam indicator; and in accordance with the extension of the sample the barrel is made to revolve by means of an arrangement which eliminates any general movement of the sample, recording that only which is due to its extension.

The author summarises the autobiography of every specimen strained to the breaking-point in the testing machine. Entering the machine in a state of internal equilibrium, its first stage is what is called in the paper one of unyielding elasticity; it extends about $\mathbf{r} / \mathbf{1 0}, 000$ of its length per ton of load, but on removal of the load remains unstrained. In its second stage the strains and stresses fluctuate, the bar yielding about 2 per cent. of its length, the strain being beyond recovery. The pencil of the indicator hesitates and almost trembles. There would seem to be a succession of local extensions in the bar, as was lately pointed out by Prof. Kennedy in this journal (NATURE, vol. xxxi. p. 504). These local extensions reduce the area locally in a higher ratio than the cohesive force increases; fracture would at once occur were it not that after a short critical interval the bar sets up increased resistance, thus entering its third stage. Stable equilibrium is restored, but the permanent strain increases in its ratio with every additional ton, and the bar may stretch 20 per cent. During the last stage the equilibrium is again unstable; the pencil steadily records a rapidly-decreasing resistance, accom. panied by a local strain which, over the part where it occurs, is very much greater than in any preceding stage. The author concludes by drawing attention to the circumstance that the apparatus records definitely the elastic limit of the material, the diagram traced gives the gross mechanical work put upon the sample, as it enables the local extension about the breaking point to be separated from the general, thus affording a means of comparing samples of different shapes ; and lastly the apparatus makes its record quite independently of the manipulation of the poise upon the steelyard.

A paper descriptive of tensile tests of iron and steel bars was read, prepared by the late Mr. Peter D. Bennett. His principal object in making these tests was to ascertain the relative effect produced on the tensile strength of a flat bar of iron or mild steel : ( I) by a hole drilled out of the bar to the required size; (2) by a hole punched $\frac{1}{8}$ inch smaller in diameter, and then drilled out to the size of the first hole; and (3) by a hole punched in the bar to the size of the drilled hole. In each of the former cases the average strength was increased per square inch of the original area across the fracture; in the third case there was a falling off in strength of nearly 20 per cent. owing to the method of perforation. The results in the first two cases were alike both for iron and mild steel, but in the third case the diminution in strength of mild steel was only 6 per cent. In another series of tests the perforated hole was filled with a rivet put in by a hydraulic machine with a pressure of thirty-one tons on the head, the results being relatively as before. The author considers these results to be due to the fact that in the drilled bar the slightly greater strain indicated was reached only along the transverse diameter of the hole, and that the strain on the metal decreased along the longitudinal diameter of the hole until it was clistributed over the whole width of the bar. Thus, at the point where it was most severely strained the metal would receive some support from the less severely strained parts adjoining.

The tests go to prove that the elongation of different testbars, all of the same length, is greatly affected by their diameter, those of larger cliameter elongating more than those of smaller 
diameter, and that as the specimens decrease in length the contraction of area at the point of fracture decreases also ; and, in consequence, the tensile strength increases when reckoned on the original area, and decreases when reckoned on the fractured area. The elongation in percentage of the original length is also very much increased in the shorter specimens, owing to the fact that the greater part of the elongation then takes place much nearer to the point of fracture, instead of being more equally distributed, as it is along the length of the longer bars.

A paper was read by Mr. A. A. Langley, descriptive of a hydraulic buffer-stop for railways, the chief advantages of which are absence of recoil after collision, continuous uniform resistance for bringing a train to rest, and absence of shock or breakage either in train or in buffer. The chief feature is the application of hydraulic resistance by the use of pistons working in horizontal cylinders filled with water, and fixed in line with the buffers of the rolling-stock.

\section{HE VALUE OF THE REFRACTION GONIO- METER IN CHEMICAL WORK ${ }^{1}$}

IMPORTAN T advances in chemistry have often been the result of new methods of research, and these have generally involved the use of new apparatus. The introluction of the balance made the greatest of revolutions in chemistry; but the therm mmeter, the blowpipe, the polariscope, and the spectroscope in its multifarious applications may also be cited as examples.

My object is to speak of the refraction goniometer or spectrometer, by which the refraction and dispersion of bodies can be measured. The construction of this instrument, and the use of it, which ought to be a part of the regular training of chemical students, may be learned from many works on physics, but it is very fully described in Glazebrook's "Optics," together with the manner in which the angular measurements are reduced to refractive indices. By means of this instrument the index of tefraction is easily obtained for liquid bodies; solids or gases require a more complicated apparatus, but those of them which can be readily dissolved in any liquid can be determined from their solutions.

The index of refraction $(\mu)$ of a body is a definite physical property, like its boiling-point, specific gravity, or solubility, and ought to form part of our knowledge of any substance. I have generally determined it for the line $\mathrm{A}$ of the spectrum of the sun; but Continental observers have usually adopted the red line of hydrogen, which is coincident with the solar line C.

The length of the spectrum-that is, the difference between the indices of refraction of extreme rays, say the lines $\mathrm{H}$ and $\mathrm{A}$, which may be taken as the measure of dispersion-is another physical property, and an equally important one.

If the index of refraction and the dispersion of a substance be accurately known, we have a double test of the purity of any specimen that may have to be examined.

As, however, the refraction varies with temperature and other circumstances, it is better to deal with the specific refraction, that is, the index, minus unity, divided by the density $\left(\frac{\mu-1}{d}\right)$. This is little, if at all, affected by pressure, heat, change of aggregate condition, mixture, solution, or, generally speaking, by chemical combination. Thus the specific refraction of water under different circumstances has been determined as follows, the observations being reduced for the line A of the spectrum :-
Water

Liquid, at $1{ }^{\circ} \mathrm{C}$

, at $48^{\circ} \mathrm{C}$.

Gas $\ldots$ with alcohol $\ldots$

Water of crystallisation
Solid $\cdots \quad \cdots \quad \ldots$
Specific refraction

... 0.329

... 0.329

... $0.331^{2}$

... 0.324

... $0.330^{3}$

$0.330^{4}$

$0.327^{5}$
The identity of the specific refraction of a body in the solid state or in solution has been frequently proved; the last instances

I Communicated to Section B of the British Association, oat the Aberdeen meeting, September 2885

4 In ammonia alum; Charles Soret.

3 Landolt ; mean of three observations.

5 In double sulphates; Topsoe and Christiansen. determined were as follows, the observations on the crystals being made by $\mathbf{M}$. Soret, and on the solutions by myself :-

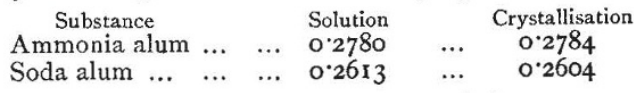

For the purpose of calculation, however, it is more convenient to adopt what Landolt denominated the refraction equivalent, that is, the specific refraction multiplied by the atomic weight $\left(P \frac{\mu-I}{d}\right)$. The refraction equivalent of water may be taken, therefore, as $0.3295 \times 18=5^{\circ} 93$. Of this, the two atoms of hydrogen may be assu ned from observations on other bodies to represent $2 \cdot 6$, leaving for the atom of oxygen 3.33 .

The specific refraction and dispersion of a body not merely gives an indication as to its purity or otherwise, but tells the quantity of the substance with which it may be mixed, if that substance is known. Thus Landolt has applied it to the quantitative analysis of mixtures, and gives examples, such as ethyl alcohol and fousel oil, ethyl alcohol and ether. I have applied it myself in the estimation of carbolic acid in disinfecting powders, by dissolving the acid out in a known quantity of alcohol, and determining the refraction and density of the solution.

In chemical investigations among organic compounds the determination of the specific refraction of the products is very valuable. Thus, in a recent investigation on the action of the copper-zinc couple on bromide of benzyl by $\mathrm{Mr}$. Tribe and myself, there were three different ways in which it led us to results which we should not otherwise have arrived at.

(I) The viscid mass which resulted from the action appeared very unpromising, but, on examining it with the prism, its specific refraction and dispersion were so high that we determined to purify it, and this led to the discovery of the new hydrocarbon benzylene.

(2) When the reaction was performed in the presence of alcohol, it seemed probable that toluene would be produced but the liquid, when heated, distilled off at $78^{\circ}$, which is the boiling-point of pure alcohol. Instead, however, of throwing the distillate away, it was examined in a hollow prism, and seen at once to be something very different. Indeed, the increased refraction and dispersion led to the belief that one-fifth of it was toluene, though that boils at $110^{\circ}$. On adding water, a liquid separated, which was proved to be toluene by its bilingpoint and density, as well as by its specific refraction and dispersion, 0.5604 and 0.0474 , agreeing sufficiently well with the known figures.

(3) On another occasion, among products of fractional distillation was a liquid which had too small a refraction to allow of its being considered a hydrocarbon. It was suspected that the low refraction might be due to oxygen or bromine; and this led to a further examination and the discovery that the liquid contained a new bromine compound.

But another important application of these physical properties is to the elucidation of the chemical structure of various bodies. A very large amount of information as to specific refraction is now at our disposal through the labours of different experimenters, not only in this country, but in Germany, Italy, Russia, Holland, and Sweden; and the whole course of recent investigations goes to show (I) that the specific refraztion of a body depends essentially on its ultimate atomic constitution; but (2) that this is modified in certain definite ways by the molecular arrangement or structure. Thus, to take an instance: sugar, $\mathrm{C}_{12} \mathrm{H}_{22} \mathrm{O}_{11}$, whether crystallised or dissolved, has the refraction equivalent of $119^{\circ} 3$. If, however, we take each atom of carbon at $5^{\circ}$, and water at $5^{\circ} 93$, we should obtain the figure $125^{\circ} \mathbf{I}$ as the calculated value. The discrepancy is far too great to be attributed to errors of experiment, and points to the fact that sugar is not, strictly speaking, a carbohydrate; that really it does not contain water, but that the hydrogen and oxygen are otherwise arranged, as chemists have concluded on other grounds. The oxygen in all hydroxyl compounds is 2.8 , according to Brihl, and this would give for sugar the theoretical value I I ${ }^{\circ} 5$, which is almost identical with the experimental number.

In a similar way it has been very fully substantiated that carbon, whenever it is in the condition which is termed "doublelinked," has the value, not of $5^{\circ} \mathrm{O}$, but of about 6.1. Hence in any compound the constitution of which is doubtful, we can tell how many carbon atoms are in this condition. Thus, to take terpene, $\mathrm{C}_{10} \mathrm{H}_{16}$. This has been the subject of much discussion among chemists, one considering it to have one pair of carbon 